Published in final edited form as:

Behav Brain Res. 2019 January 01; 356: 250-256. doi:10.1016/j.bbr.2018.08.028.

\title{
Dopamine $D_{1}$ and $D_{2}$ Receptor Antagonism During Development Alters Later Behavior in Zebrafish
}

\author{
Anthony N. Oliveri ${ }^{1}$ and Edward D. Levin ${ }^{1,2}$ \\ ${ }^{1}$ Department of Pharmacology and Cancer Biology, Duke University School of Medicine, Durham, \\ NC, USA, 27710 \\ ${ }^{2}$ Department of Psychiatry and Behavioral Sciences, Duke University School of Medicine, \\ Durham, NC, USA, 27710
}

\begin{abstract}
This study sought to examine the long-term behavioral impacts of dopamine $\mathrm{D}_{1}$ and $\mathrm{D}_{2}$ receptor antagonism during development in zebrafish (Danio rerio). Zebrafish embryos of both the $\mathrm{AB}^{*}$ and 5D strains were exposed via immersion to either the $\mathrm{D}_{1}$ receptor antagonist SCH-23390 or the $\mathrm{D}_{2}$ receptor antagonist haloperidol, at either 0.5 or $1.5-\mu \mathrm{M}$, from $5 \mathrm{~h}$ post-fertilization to 5 days post-fertilization. Aquarium water served as a control. Fish were then either tested as larvae on day 6 post-fertilization on a light/dark locomotor assay, or were grown to adulthood and tested on a behavioral battery that included assays for novel environment exploration, startle habituation, social affiliation, and predator escape (AB* strain only). Overall, developmental exposure to dopamine $\mathrm{D}_{1}$ and $\mathrm{D}_{2}$ receptor antagonists caused clear effects in larval locomotor behavior, driving hyperactivity in dark phases and hypoactivity in light phases. Additionally, control fish from the two strains were significantly different from each other $(\mathrm{p}<0.05)$ with the $\mathrm{AB}^{*}$ fish being more active than SD during the dark periods of the test.. In the adult behavioral battery, developmental exposure to $1.5-\mu \mathrm{M}$ of the $\mathrm{D}_{1}$ antagonist $\mathrm{SCH}-23390$ significantly reduced activity $(\mathrm{p}<0.05)$ in the predator escape assay. Despite the fact that embryonic exposure to $\mathrm{D}_{1}$ and $\mathrm{D}_{2}$ receptor antagonists caused clear behavioral alterations in larval activity there were much more subtle effects persisting into adulthood.
\end{abstract}

\section{Keywords}

Zebrafish; Dopamine; Development; Sensorimotor; Shoaling; Predatory Escape

\section{Introduction}

Dopamine signaling is known to be integral to normal neurobehavioral development [1-3]. In rats, blocking $\mathrm{D}_{2}$ receptors with the drug haloperidol during development leads to

Communicating Author: Edward D. Levin, Ph.D., Department of Psychiatry and Behavioral Sciences, Box 104790, Duke University Medical Center, Durham, NC 27710, USA, edlevin@ duke.edu, Phone: 1-919-681-6273; Fax: 1-919-681-3416.

Publisher's Disclaimer: This is a PDF file of an unedited manuscript that has been accepted for publication. As a service to our customers we are providing this early version of the manuscript. The manuscript will undergo copyediting, typesetting, and review of the resulting proof before it is published in its final citable form. Please note that during the production process errors may be discovered which could affect the content, and all legal disclaimers that apply to the journal pertain. 
reductions in brain weight [4] and increased dopamine receptor binding throughout the lifespan [5]. Behaviorally, postnatal exposure to haloperidol in mice leads to hyperactivity [6], while prenatal exposure in rodents produces a decrease in normal circling behavior in the offspring, a deficit that can be corrected with later acute administration with the same drug [7]. Additionally, rats dosed developmentally with the $\mathrm{D}_{2 / 3}$ receptor agonist quinpirole exhibited reductions in spatial learning later in life [8]. Similarly, prairie vole pups exposed to the $\mathrm{D}_{1}$ receptor agonist SKF-38393 (though not to a $\mathrm{D}_{1}$ antagonist or $\mathrm{D}_{2}$ agonist or antagonist) during development showed reduced pair-bonding as adults, without altering $\mathrm{D}_{1}$ or $\mathrm{D}_{2}$ receptor binding [9]. The later behavioral effects of developmental exposure to haloperidol may be clearest when the exposure occurs during critical periods of striatal development [10] and although the mechanisms driving these lasting behavioral effects of developmental dopamine receptor modulation remain largely unknown, it has been shown that exposure to haloperidol in critical stages of development alters the formation and structure of dendrites in multiple brain regions in mice [11].

The use of zebrafish to study various facets of neurodevelopment, including the role of dopamine receptors, is a rapidly expanding field. The components of the dopaminergic system of zebrafish are largely conserved compared to other vertebrates, including homologs of both $\mathrm{D}_{1^{-}}$and $\mathrm{D}_{2}$-receptor subtypes [12]. Structurally, zebrafish do lack a midbrain population of dopamine neurons, but instead possess a large grouping of them in the telencephalon, although whether this population is analogous to the midbrain population in mammals is still unclear [12]. A number of studies have examined the effects of specific dopamine receptor agonism and antagonism on behavior in zebrafish. Zebrafish larvae dosed acutely with $D_{1}$ and $D_{2}$ antagonists display marked hypoactivity $[13,14]$ while agonism of either receptor subtype produces hyperactivity [14]. In adults, acute dosing with dopamine receptor antagonists improved spatial learning and memory, while fish dosed with dopamine receptor agonists showed impaired performance [15]. Dopamine D4 receptors have been found to play a key role in the signaling necessary for the ontogeny of locomotion [16]. However, the question of long-term consequences of the developmental antagonism of specific dopamine receptors in zebrafish is a relatively unexplored one. One previous study in our lab exposed zebrafish during the first five days post-fertilization to the dopamine reuptake inhibitor methylphenidate, and found persistent changes in exploratory behavior and spatial discrimination accuracy [17], suggesting that the zebrafish model is sensitive to the neurobehavioral effects following alterations in dopamine signaling during development.

A full characterization of the neurobehavioral consequences following the interruption of dopamine signaling during development in zebrafish will likely be important for the elucidation of the effects of various compounds in zebrafish assays for developmental neurotoxicity. As zebrafish continue to be developed as screens for neuropharmacology and neurotoxicology, knowledge of the effects of dopamine system antagonism during development on zebrafish behavior across the lifespan will be critical. To that end, this study sought to characterize the behavioral effects across the lifespan following developmental exposures to dopaminergic antagonist. Zebrafish of the $\mathrm{AB}^{*}$ strain were exposed to either the $\mathrm{D}_{1}$ receptor antagonist $\mathrm{SCH}-23390$ or the $\mathrm{D}_{2}$ receptor antagonist haloperidol were exposed for the first 5 days post-fertilization, and were subsequently tested on a battery of behavioral assays across both larval and adult life stages. Subsequently, we repeated these 
exposures and the larval behavioral assay with zebrafish of another strain, 5D, in order to begin evaluating whether different strains of zebrafish may react differentially to developmental disruption of dopaminergic systems.

\section{Methods}

\section{Animal Housing and Husbandry}

Zebrafish ( $\mathrm{AB}^{*}$ strain) were bred from a colony originating with progenitors obtained from the Zebrafish International Resource Center (ZIRC, Eugene, OR, USA). Zebrafish of the 5D strain were bred from a colony originating from Oregon State University. Breeding tanks of $\mathrm{N}=12-15$ were maintained with a male to female ratio of 2:1. Eggs were collected via intank inserts approximately 1-2 hours after the lights-on phase of a 14:10 h light:dark cycle. Eggs from approximately 6 such tanks were combined and rinsed with 10,000× diluted solution of bleach for 1 minute, followed by 3 likewise rinses in fresh aquarium water. Eggs were inspected under a dissection microscope and unfertilized or otherwise abnormal eggs were discarded. Approximately 5-h post-fertilization, eggs $(\mathrm{N}=50)$ in two exposure replicates were randomly distributed into glass Petri dishes corresponding to differing exposures, and placed in an incubator held at $29^{\circ} \mathrm{C}$ and illuminated with an identical 14:10 light cycle until 6 days post-fertilization.

Fish aged 6 days post-fertilization and older were housed in 3L tanks on a circulating aquarium rack system (Aquatic Habitats/Pentair Aquatic Eco-Systems, Apopka, FL, USA). Aquarium water was made from a mixture of sea salt (Instant Ocean, 0.5 parts per thousand) and buffer (Seachem Neutral Regulator, 2.5-g/ $\mathrm{L} \mathrm{H}_{2} \mathrm{O}$ ) dissolved in de-ionized water, and was maintained at $26^{\circ} \mathrm{C}$. Water chemistry, salinity, and temperature were monitored biweekly. Fish were fed twice per day: a suspension of 24-hour-old brine shrimp raised inhouse (origin Brine Shrimp Direct, Ogden, UT, USA) in the morning, and solid food (TetraMin Tropical Flakes, Blacksburg, VA, USA) in the evening. Younger fish (until 3-4 weeks postfertilization) were supplemented with smaller-particle solid food (Brine Shrimp Direct Golden Pearl). All adult behavioral testing was conducted between 11:00 AM (2-h post lights-on) and 6:00 PM, with testing time counterbalanced across experimental groups. On days of behavioral testing the evening feeding was withheld until testing was complete. All larval testing was run between 3:00 PM and 5:00 PM.

\section{Larval Motility Assay}

At 5 hours post-fertilization, zebrafish eggs of both $\mathrm{AB}^{*}$ and 5D strains were placed into 96well plates with meshed plastic inserts in $0.5-\mathrm{ml}$ of solutions of SCH-23390 (Sigma-Aldrich, St. Louis, MO, USA) or haloperidol (Abbott Labs, Abbott Park, Il, USA) at either 0.5 or $1.5 \mu \mathrm{M}$. These concentrations were based on the results of pilot studies to be subthreshold for causing increased lethality and morphological deformities. Aquarium water served as control, and all solutions were renewed every 24 hours, through 5 days post-fertilization. These solutions were renewed every 24 hours, through 5 days post-fertilization. Hatched larvae were then placed into fresh aquarium water for $24 \mathrm{~h}$, at which point they were examined under a dissecting microscope. Larvae exhibiting arrested development or malformations were discarded. Exposure conditions were all represented within each plate 
and across multiple plates. Plates were then returned to the incubator for an hour before being placed into a DanioVision ${ }^{\mathrm{TM}}$ lightbox running EthoVision $\mathrm{XT}^{\circledR}{ }^{\circledR}$ tracking software (Noldus, Wageningen, The Netherlands). After an initial 10-min acclimation period in the dark ( $0 \%$ illumination), locomotor activity was tracked during a paradigm composed of 2 cycles of 10 -min at $100 \%$ illumination (5,000 lux) and 10-min at $0 \%$ illumination. An infrared camera tracked larval locomotion across the 40-min trial. EthoVision $\mathrm{XT}^{\circledR}$ was used to calculate the average distance moved in $\mathrm{cm}$ per minute for each subject.

\section{Adult Behavioral Test Battery}

Zebrafish eggs of the $\mathrm{AB}^{*}$ strain were dosed as in the larval motility assay described above, with the exception that the eggs were placed into $40 \mathrm{ml}$ of solution in glass petri dishes instead of 96-well plates. 6-day-old larvae distinct from those used in the larval motility assay were transferred to the aquarium rack systems and allowed to age normally to 12 weeks of age ( $\mathrm{N}=16-23$ per exposure condition over two exposure replicates). Over approximately the following two weeks, the adult fish were run through a series of behavioral tests assessing various behavioral and cognitive functions.

\section{Adult Novel Tank Diving Test}

Adult fish were assessed for novel environment exploration in a test described previously $[18,19]$. Briefly, fish were placed into $1.5 \mathrm{~L}$ tanks filled $10-\mathrm{cm}$ high with aquarium water. A camcorder feeding into EthoVision $\mathrm{XT}^{\circledR}$ software was used to track the position of the fish across a 5-min trial, and to calculate both the average distance moved in $\mathrm{cm}$ per minute and the average distance from the floor of the tank in $\mathrm{cm}$ per min of the test.

\section{Adult Sensorimotor Startle Response Habituation}

Habituation to a startling stimulus was tested in adult fish via a protocol used previously [19-21]. Adult fish were placed into 40-ml of water in one of 8 translucent plastic cups (5.7$\mathrm{cm}$ in diameter) arranged in a $4 \times 2$ array. Below each cup was a centrally located push solenoid that could be controlled to deliver a sudden tap to the bottom of the cup. A camcorder positioned above the cups was used to record the locomotion of the fish in all 8 cups at once. Following a 5-min acclimation period after the fish were placed in the cups, 10 taps were delivered once per minute over $10 \mathrm{~min}$. EthoVision $\mathrm{XT}^{\circledR}$ software was used to control the solenoids and to calculate the average distance moved in $\mathrm{cm}$ during the 5 seconds preceding and following each of the 10 taps.

\section{Adult Social Affiliation}

The third test in the behavioral battery, an assessment of social affiliation, utilized a type of behavioral response called shoaling, in which a zebrafish will interact with a group of conspecific fish (a shoal) as described previously [19]. Adult fish were singly isolated in 1.5L tanks surrounded by opaque dividers for 1 hour. After the hour they were placed in a large transparent rectangular tank $45-\mathrm{cm}$ long by $13-\mathrm{cm}$ wide by $12.5-\mathrm{cm}$ deep. On both ends of the tank was positioned a computer monitor for stimulus display. The fish was recorded using a digital video recorder placed above the tank. During the first minute of the 6-minute test, each monitor screen displayed a background of static ovals approximately the size of an 
adult zebrafish and displaying the pattern and colors of a typical zebrafish. At the end of the first minute, one of the two monitors began to display a video recording of a zebrafish shoal for the remaining 5 minutes. The side of the tank displaying the shoal video was counterbalanced among exposure groups. EthoVision $\mathrm{XT}^{\circledR}$ was used to control the two displays, to record the locomotion of the fish, and to calculate both the average distance traveled in $\mathrm{cm}$ per minute and the average distance from the tank wall through which the shoal video was visible in $\mathrm{cm}$ for each subject across the $6 \mathrm{~min}$ of the test.

\section{Adult Predator Stimulus Escape}

The final test involved an assessment of escape and avoidance behavior following exposure to a predator-like stimulus. Adult fish were placed in a $1.5 \mathrm{~L}$ tank facing a computer monitor. The 5-min test involved an initial minute of acclimation, followed by 2 rounds of a cycle including a 1-min presentation of a small $(1.3-\mathrm{cm}$ diameter) circle appearing in the center of the screen, growing to $30.5 \mathrm{~cm}$ in diameter, disappearing, and repeating, to simulate a larger fish swimming towards the tank, followed by 1 min of a blank screen. The test was performed first with a blue dot that grew slowly (4-s), and again several days later with a red dot that grew quickly (1-s). A camcorder positioned above the tank tracked the fish. EthoVision $\mathrm{XT}^{\circledR}$ was used to control the display, to record the fish's activity, and to calculate both the average distance traveled in $\mathrm{cm}$ per minute and the average distance from the tank wall through which the screen was visible in $\mathrm{cm}$ for each subject over the 5-min [19].

\section{Statistical Analysis}

The dependent measures corresponding to each assessment are described above. All statistical analyses were performed with SuperANOVA/Statview (SAS, Cary, NC, USA). Type 1 error rate (a) was set at 0.05 for all tests. A mixed design repeated measures of analysis (RMANOVA) with antagonist exposure and dose as the between subject factor and tap number, session minute, or time condition as the repeated measures was used for all behavioral tests. Fisher's least significant difference was used for all post-hoc analyses. A Huynh-Feldt adjustment was used to control for possible deviations from sphericity. Log transformations of the raw data were performed (as noted) when the distribution of the data was positively or negatively skewed. Interactions $p<0.10$ were followed by tests of simple main effects as recommended by Snedecor and Cochran [22]. In final analyses $\mathrm{p}<0.05$ (twotailed) was always set as threshold for statistical significance.

\section{Results}

\section{Embryonic Survival}

Viability and survival of embryos was tracked daily during the dosing period of the first 5 days post-fertilization. The majority of dead or nonviable eggs were identified within the first 24-h after plating and were likely due to misidentification of fertilized or healthy eggs at the time of egg selection plating at $5 \mathrm{hpf}$. These eggs regularly accounted for approximately $30 \%$ of the eggs plated and death across the remainder of the exposure window was negligible. At 6 days post-fertilization, the hatched larvae were examined for physical malformations under a dissecting scope. Abnormal spinal curvature or edema of the pericardium or yolk sac qualified an embryo for exclusion from all further testing. 
Preliminary experiments had identified threshold doses producing these malformations, and the exposure doses and conditions selected for this study did not produce significant increases in physical abnormalities.

\section{Larval Motility Assay}

Both the $\mathrm{AB}^{*}$ and $5 \mathrm{D}$ strains of zebrafish were tested in the light-dark larval motility assay. The main effect of the repeated measure of lighting condition $(F(1,266)=995.75 p<0.001)$ on locomotor activity was significant. Effects of the between subjects factors of zebrafish strain $(\mathrm{F}(1,266)=4.60, \mathrm{p}<0.05)$, and drug treatment $(\mathrm{F}(4,266)=11.30, \mathrm{p}<0.001)$ on locomotor activity were all observed as well. Additionally, there were significant interactions between lighting condition $\mathrm{x}$ zebrafish strain $(\mathrm{F}(1,266)=44.88, \mathrm{p}<0.001)$ and zebrafish strain $\mathrm{x}$ drug treatment $(\mathrm{F}(4,266)=3.70, \mathrm{p}<0.01)$, also there was an interaction of lighting condition $\mathrm{x}$ drug treatment $(\mathrm{F}(4,266)=2.36, \mathrm{p}=0.054)$ that prompted separate analysis of drug effects in each strain (Fig. 1). In the light phase, the simple main effect of drug treatment showed that zebrafish exposed to both doses of SCH-23390 were significantly hypoactive compared to control larvae $(\mathrm{p}<0.05)$. A significant interaction of zebrafish strain $\mathrm{x}$ drug treatment within the dark phase $(\mathrm{F}(4,266)=5.07, \mathrm{p}<0.001)$ prompted analyses of the simple main effects of treatment within each strain. In the AB* strain fish the $1.5-\mu \mathrm{M}$ dose of SCH-23390 significantly reduced activity in the dark phase, that treatment had no significant effect in fish of the 5D strain. Instead, fish exposed to the other three treatment conditions ( 0.5 and $1.5-\mu \mathrm{M}$ haloperidol and $0.5-\mu \mathrm{M} \mathrm{SCH}-23390$ ) had significantly increased activity in the dark condition (Fig. 1A-B). Finally, post-hoc analyses of a significant main effect of strain on activity of control fish in the dark phase $(F(1,76)=48.83, p<0.001)$ revealed that control fish of the $\mathrm{AB}^{*}$ strain swam significantly more in the dark compared to fish of the $5 \mathrm{D}$ strain $(\mathrm{p}<0.05)$ (Fig. 2).

\section{Adult Novel Tank Diving Test}

The zebrafish of the AB* strain were also tested in the adult behavioral test battery. In the novel tank diving test, there were significant main effects of minute of the test with both the distance from the bottom of the tank $(F(4,452)=31.28$, $\mathrm{p}<0.001)$ (Fig. $3 \mathrm{~A})$ and on total locomotor activity $(\mathrm{F}(4,452)=58.43$, $\mathrm{p}<0.001)$ (Fig. 3B). There were no significant main effects of treatment, although an interaction of treatment and minute on distance from the bottom of the testing tank $(\mathrm{F}(16,452)=1.537, \mathrm{p}=0.08)$ prompted follow up analyses examining the linear trends of the dive response over time. However, there were no significant effects of treatment on the linear trend across the testing time.

\section{Adult Sensorimotor Startle Response and Habituation}

Data from the startle habituation task were log transformed prior to analysis to reduce skewedness. The ten startle stimuli were averaged into five blocks of two for statistical analysis. Presentation of the tap stimulus produced a significant main effect of activity prevs. post-tap on locomotor activity $(\mathrm{F}(1,113)=165.17$, $\mathrm{p}<0.001)$, with the fish moving significantly more in the $5 \mathrm{~s}$ following the tap stimuli compared to the $5 \mathrm{~s}$ preceding the stimulus. The block number of sequential taps was also significant $(F(4,452)=4.97$, $\mathrm{p}<0.001)$, with activity decreasing over time. A significant interaction of swimming activity 
pre-vs. post stimulus presentation by block number was also observed $(\mathrm{F}(4,452)=12.92$, $\mathrm{p}<0.001$ ). Drug treatment produced no significant effects on the tap startle test (Fig. 4).

\section{Adult Social Affiliation}

In the social affiliation test the two measures taken were average distance from the screen with the shoaling stimulus and swimming speed $(\mathrm{cm} / \mathrm{min})$. No drug treatment effects were observed in either the distance to shoal (Fig. 5A) or swimming speed (Fig. 5B). There was a significant main effect of time on locomotor activity in the social affiliation task $(\mathrm{F}(4,388)=15.07$, $\mathrm{p}<0.001)$, with swimming speed increasing as the session progressed (Fig. $5 \mathrm{~B})$.

\section{Adult Predator Stimulus Escape}

In the predator escape paradigm, two measures were taken, average distance from the visual stimulus screen and swimming speed $(\mathrm{cm} / \mathrm{sec})$. the distance of the fish from the tank wall through which the computer screen was visible was significantly increased during the minutes consisting of the presentation of either the red or blue stimulus, compared to those during which the control image was displayed $(F(1,166)=266.02$, $p<0.001)$. Stimulus presentation also significantly increased locomotor activity $(F(1,166)=39.44, p<0.001)$. A significant main effect of drug treatment was seen with locomotor activity $(F(4,166)=4.28$, $\mathrm{p}<0.005)$. Fish exposed to the $1.5-\mu \mathrm{M}$ dose of $\mathrm{SCH}-23390$ moved significantly less over the course of the test compared to controls ( $\mathrm{p}<0.05$ ) (Fig. 6).

\section{Discussion}

Here, we show that early developmental antagonism of dopamine receptors can alter zebrafish behavior during the larval stage immediately following exposure and persisting into adult life stages. In the larval locomotor test, developmental exposure to the antagonists generally produced hyperactivity in the dark phase and hypoactivity in the light. Furthermore, the reduction of activity in the light phase appeared more sensitive to developmental exposure to the $\mathrm{D}_{1}$ receptor antagonist, $\mathrm{SCH}-23390$, while the larvae exposed to the $\mathrm{D}_{2}$ receptor antagonist haloperidol showed clearer hyperactivity in the dark. The dopamine antagonists drove differential effects across the two strains tested, $\mathrm{AB}^{*}$ and 5D, partially due to a large difference in activity in the dark in the control fish of each strain, with the patterns observed in the dark with both strains combined being drive almost entirely by effects seen in the 5D strain alone. In adult fish, developmental exposure to the dopamine antagonists decreased locomotor activity in the predator escape testing paradigm, with fish exposed to the highest dose of SCH-23390 showing the most significant reduction. Conversely, developmental exposure to either antagonist had no effect on adult behavior in the novel environment exploration, startle habituation, and the social affiliation tests.

In this study, developmental exposure to selective dopamine receptor antagonists appeared to drive more marked behavioral changes in larval fish than compared to adults. This may reflect a specificity in the behavioral domains impacted via developmental dopamine antagonism. The behavior in adult zebrafish most significantly affected by the exposures was in the predator escape assay, where treated fish displayed reduced locomotion across the 
duration of the test. Of all the adult behavioral assays, the predator escape test also most closely resembles the larval locomotor assay - a measure of locomotor response across intermittent presentation of a visual stimulus (changing light conditions with larval fish, and the growing dot representing a predator in adults). Notably, it was the $\mathrm{AB}^{*}$ larvae exposed to the $1.5 \mu \mathrm{M}$ dose of SCH-23390 that exhibited a significant decrease in activity in the dark, while larvae of both strains appeared equally susceptible to the effects of either SCH-23390 dose in the light. Later, in $\mathrm{AB}^{*}$ adults, fish exposed to the same higher dose of the $\mathrm{D}_{1}$ receptor antagonist showed a significant decrease in swimming activity in the predator escape assay. The conserved drug- and dose-sensitivity across the larval locomotor and adult predator avoidance assays further supports the possibility of the predator escape assay being the closest to the larval test in the adult battery. In this way, it may be that the other adult behavioral domains were unaffected because the lasting neurobiological changes following the administration of the antagonists were specific for this type of locomotor response to visual stimuli, leaving exploratory or social behavior intact, for example. The startle habituation test, while consisting of locomotor responses to repeated stimuli, involves a different modality of stimulus presentation.

Another possible explanation for the relatively prominent behavioral effects seen in larval fish as opposed to adults, one not mutually exclusive with the discussion of behavioral domains above, is that the developmental exposure paradigm used in this study across the first five days post-fertilization, created a state of acute adaptation in the dopaminergic systems of the developing larvae. This may be supported by the fact that the trends seen here, of activity increasing in the dark and decreasing in the light, is the opposite of what has been observed following acute administration with these compounds [14], suggesting that the dopaminergic system compensated via upregulation for the chronic receptor antagonism across the first five days post-fertilization which, in turn, may have resulted in lingering abnormal behavior indicative of increased dopaminergic activity 24 hours later when the larvae were tested in the locomotor assay. This effect would not be unlike the withdrawal seen after regular use of psychoactive drugs. Note that this hypothesis does not preclude lasting developmental changes in the zebrafish brain; it is certainly possible that the behavioral effects were clearer in larvae due to the stillpresent "withdrawal" from the antagonists, and that simultaneously the antagonism (or the adaptive response to the antagonism, or both) were creating long-lasting developmental abnormalities that drove the subtler and more selective effects seen in adults.

There may be accommodation between $\mathrm{D}_{1}$ and $\mathrm{D}_{2}$ receptors when either one is blocked during development where $\mathrm{D}_{2}$ show a compensatory response when $\mathrm{D}_{1}$ receptors are blocked and visa versa. Further research with added study of the effect of blocking both D1 and $\mathrm{D} 2$ receptors would provide added information concerning this possible compensation across the different subtypes of dopamine receptors.

Evaluating whether developmental antagonism of dopamine receptors creates lasting dysfunction in dopaminergic systems also requires additional testing on behavioral dimensions not tested in the current study. For example, spatial learning and discrimination has been shown to be impacted by acute dopamine agonism and antagonism [15], and performance on a spatial learning task has been shown to correlate with DOPAC levels [23]. 
Alternatively, conditioned place preference, a behavioral assay long associated with dopaminergic reward pathways, has been shown to be similarly sensitive to modulation of dopamine signaling in zebrafish [24]. It is possible that other neurotransmitter systems may be impacted and driving any lasting behavioral effects, as acute dopaminergic antagonism in adult zebrafish decreases the levels of not only dopamine and its metabolite DOPAC, but also serotonin and its metabolite 5HIAA [25]. This might help explain why previous research has shown social affiliation in adult zebrafish to be sensitive to acute dopamine receptor antagonism [26] but no deficits in shoaling behavior were seen in the current study following developmental exposure.

It is important to remember that all drugs have diverse effects, $\mathrm{SCH}-23390$ and haloperidol included. Dopaminergic receptors are divided into two families, $\mathrm{D}_{1}$-like which includes $\mathrm{D}_{5}$ as well as $\mathrm{D}_{1}$ and $\mathrm{D}_{2}$-like which includes $\mathrm{D}_{3}$ and $\mathrm{D}_{4}$ as well as $\mathrm{D}_{2}$. SCH-23390 has antagonistic effects at dopamine $\mathrm{D}_{5}$ receptors as well as $\mathrm{D}_{1}$ receptors. In a similar manner haloperidol has antagonistic effects at $\mathrm{D}_{3}$ and $\mathrm{D}_{4}$ as well as $\mathrm{D}_{2}$ receptors. $\mathrm{SCH}-23390$ and haloperidol also have actions at non-dopaminergic receptors. SCH23390 acts at serotonin 1c receptors [27] and haloperidol acts at a 1 adrenergic receptors [28].

Finally, the findings in this study from the larval locomotor assay contribute to a growing literature regarding neurobehavioral differences between zebrafish strains. As seen here, baseline behavioral responses were found to vary among zebrafish strains in tests of locomotor behavior [29-32], inhibitory avoidance behavior [33], and social affiliation [34]. Additionally, strains were found to react differentially to acute administration of dopamine receptor antagonists [26], to have different levels of dopamine and DOPAC. [34, 35], and to differentially express the genes for the $\mathrm{D}_{1}$ dopamine receptor [35]. These differences between strains may help explain the differences in control larval behavior and the differential responses to dopamine antagonist exposure seen between the $\mathrm{AB}^{*}$ and $5 \mathrm{D}$ strains observed in this study. Interestingly, the larvae of the $\mathrm{AB}^{*}$ strain appeared sensitive to the effects of developmental exposure to SCH-23390 alone, with no significant effects of developmental dosing with haloperidol observed. In the 5D strain, both drugs produced significant effects in the dark phases, with fish exposed to haloperidol showing an even greater effect than seen with SCH-23390. Interestingly, in these 5D larvae we observed greater effects in the dark phases with larvae exposed to the lower dose of SCH-23390, suggesting a differential sensitivity to $\mathrm{D}_{1}$ receptor antagonism in the $5 \mathrm{D}$ strain compared to the $\mathrm{AB}$ strain. It might be that an increased sensitivity in the 5D strain manifested as a greater compensatory response than produced in the $\mathrm{AB}^{*}$ strain in the case of the lower dose of SCH-23390, whereas this increased sensitivity then began to induce enough dysfunction that a decrease in locomotion (compared to the lower dose) was seen at the higher dose, bringing the activity level back down to a level similar to control fish. Future studies seeking to elaborate on the differential responses by strain seen here should seek to characterize a broader dose-response curve to elaborate on this apparent non-monotonic dose effect. Additionally, this suggests that establishing whether developmental antagonism of dopamine receptors in the 5D strain could lead to behavioral effects in adult zebrafish would be an important follow-up to this study. 
We have found that administration of selective dopamine receptor antagonists during embryonic development alters later behavior in zebrafish. This study suggests that zebrafish can be a sensitive screen for developmental neurobehavioral toxicity associated with pharmaceuticals, drugs of abuse, and environmental toxicants that are suspected to impact dopaminergic systems. In particular, dopamine $\mathrm{D}_{1}$ and $\mathrm{D}_{2}$ systems appear to play important roles in brain development, inasmuch as antagonist treatment during development cause both short and long-term behavioral effects. These results imply that other drugs and chemicals that disrupt dopamine systems could have persisting behavioral effects as well. Our findings also show the importance of broad-based behavioral testing, as teratology might frequently be behavior-specific and may be missed when only testing one or two behavioral domains. Finally, because of behavioral and neurochemical differences among zebrafish strains, more comprehensive strain characterizations will be integral to ensuring replicable and translatable studies in zebrafish.

\section{Acknowledgements}

This research was supported by the US-EPA Star grant 83554101 and the NIEHS Superfund Research Program (ES010356)

\section{References}

1. Missale C, et al., Dopamine receptors: from structure to function. Physiological Review,1998 78: p. 189-225.

2. Riddle R and Pollock JD, Making connections: The development of mesencephalic dopaminergic neurons. Developmental Brain Research, 2003 147: p. 3-21. [PubMed: 14741747]

3. Gatzke-Kopp LM, The canary in the coal mine: The sensitivity of mesolimbic dopamine to environmental adversity during development. Neuroscience and Biobehavioral Reviews, 2011 35: p. 794-803. [PubMed: 20888857]

4. Williams R, et al., Prenatal haloperidol exposure: effects on brain weights and caudate neurotransmitter levels in rats. Brain Research Bulletin, 1992 29(3-4): p. 449-58. [PubMed: 1356601]

5. Moon SL, Prenatal haloperidol alters striatal dopamine and opiate receptors. Brain Research, 1984 323: p. 109-113. [PubMed: 6098328]

6. Dallemagne G and Weiss B, Altered adult behavior of mice following postnatal treatment with haloperidol. Pharmacology, Biochemistry and Behavior, 1982 16: p. 761-767.

7. Wolansky MJ, et al., Postnatal haloperidol eliminates the deficit in circling behavior produced by prenatal exposure to the same drug. Neurotoxicology \& Teratology, 2004 26(4): p. 561-9. [PubMed: 15203178]

8. Vorhees CV, et al., Developmental treatment with the dopamine D2 / 3 agonist quinpirole selectively impairs spatial learning in the Morris water maze. Neurotoxicology and Teratology, 2009 31: p. 110. [PubMed: 18930132]

9. Hostetler CM, et al., Neonatal exposure to the D1 agonist SKF38393 inhibits pair bonding in the adult prairie vole. Behavioural Pharmacology, 2011 22: p. 703-710. [PubMed: 21918384]

10. Soiza-Reilly M and Azcurra JM, Developmental striatal critical period of activity-dependent plasticity is also a window o susceptibility for haloperidol induced adult motor alterations. Neurotoxicology and Teratology, 2009 31: p. 191-197. [PubMed: 19336253]

11. Frost DO, et al., Early exposure to haloperidol or olanzapine induces long-term alterations of dendritic form. Synapse, 2010 64(3): p. 191-9. [PubMed: 19862684]

12. Panula P, et al., The comparative neuroanatomy and neurochemistry of zebrafish CNS systems of relevance to human neuropsychiatric diseases. Neurobiologic Disease, 2010 40: p. 46-57. 
13. Giacomini NJ, et al., Antipsychotics produce locomotor impairment in larval zebrafish. Neurotoxicology \& Teratology, 2006 28(2): p. 245-250. [PubMed: 16527449]

14. Irons TD, et al., Acute administration of dopaminergic drugs has differential effects on locomotion in larval zebrafish. Pharmacology, Biochemistry \& Behavior, 2013 103(4): p. 792813.

15. Naderi M, et al., Dopamine receptors participate in the acquisition and consolidation of latent learning of spatial information in zebrafish (Danio rerio). Progress in Neuropsychopharmacology and Biological Psychiatry, 2016 67: p. 21-30.

16. Lambert AM, Bonkowsky JL, and Masino MA, The Conserved Dopaminergic Diencephalospinal Tract Mediates Vertebrate Locomotor Development In Zebrafish Larvae DDT Mediates Zebrafish Locomotor Development. Journal of Neurosciience, 2012 32(39): p. 13488-13500.

17. Levin ED, et al., Persistent behavioral impairment caused by embryonic methylphenidate exposure in zebrafish. Neurotoxicology and Teratology, 2011 33: p. 668-673. [PubMed: 21741476]

18. Bencan $\mathrm{Z}$ and Levin ED, The role of alpha7 and alpha4beta2 nicotinic receptors in the nicotineinduced anxiolytic effect in zebrafish. Physiology \& Behavior, 2008 95(3): p. 40812.

19. Oliveri AN, Bailey JM, and Levin ED, Developmental exposure to organophosphate flame retardants causes behavioral effects in larval and adult zebrafish. Neurotoxicology and Teratology, 2015 52: p. 220-227. [PubMed: 26344674]

20. Eddins D, et al., Zebrafish provide a sensitive model of persisting neurobehavioral effects of developmental chlorpyrifos exposure: comparison with nicotine and pilocarpine effects and relationship to dopamine deficits. Neurotoxicology \& Teratology, 2010 32(1): p. 99-108. [PubMed: 19268529]

21. Sledge D, et al., Critical duration of exposure for developmental chlorpyrifos-induced neurobehavioral toxicity. Neurotoxicology and Teratology, 2011 33: p. 742-751. [PubMed: 21745564]

22. Snedecor GW and Cochran WG, Statistical Methods. 1967, Ames, Iowa: Iowa State University Press.

23. Eddins D, et al., Nicotine effects on learning in zebrafish: the role of dopaminergic systems. Psychopharmacology, 2009 202(1-3): p. 103-109. [PubMed: 18716760]

24. Darland T, et al., Sulpiride, but not SCH23390, modifies cocaine-induced conditioned place preference and expression of tyrosine hydroxylase and elongation factor 1a in zebrafish. Pharmacology, Biochemistry and Behavior, 2012 103: p. 157-167.

25. Tran S, et al., Differential effects of acute administration of SCH-23390, a D1 receptor antagonist, and of ethanol on swimming activity, anxiety-related responses, and neurochemistry of zebrafish. Psychopharmacology, 2015 232: p. 3709-3718. [PubMed: 26210378]

26. Scerbina T, Chatterjee D, and Gerlai R, Dopamine receptor antagonism disrupts social preference in zebrafish: a strain comparison study. Amino Acids, 2012 43: p. 2059-2072. [PubMed: 22491827]

27. Nicklaus KJ, McGonigle P, and Molinoff P, [3H]SCH 23390 labels both dopamine-1 and 5hydroxytryptamine1c receptors in the choroid plexus. The Journal Pharmacology and Experimental Therapeutics, 1988 247(1): p. 343-348.

28. Richelson E and Souder T, Binding of antipsychotic drugs to human brain receptors focus on newer generation compounds. Life Sciences, 2000 68(1): p. 29-39. [PubMed: 11132243]

29. de Esch C, et al., Locomotor activity assay in zebrafish larvae: Influence of age, strain and ethanol. Neurotoxicology and Teratology, 2012 34: p. 425-433. [PubMed: 22484456]

30. Gao Y, et al., Computational classification of different wild-type zebrafish strains based on their variation in light-induced locomotor response. Comput. Biol. Med, 2016 69: p. 1-9. [PubMed: 26688204]

31. Lange M, et al., Inter-individual and inter-strain variations in zebrafish locomotor ontogeny. PLoS One, 2013 8: p. e70172. [PubMed: 23950910]

32. Vignet G, et al., Systematic Screening of Behavioral Responses in Two Zebrafish Strains. Zebrafish, 2013 10(3): p. 365-375. [PubMed: 23738739]

33. Gorissen M, et al., Differences in inhibitory avoidance, cortisol and brain gene expression in TL and AB zebrafish. Genes. Brain and Behavior, 2015 14: p. 428-438. 
34. Mahabir S, et al., Maturation of shoaling in two zebrafish strains: A behavioral and neurochemical analysis. Behavioural Brain Research, 2013 247: p. 1-8 [PubMed: 23518435]

35. Pan Y, Chatterjee D, and Gerlai R, Strain dependent gene expression and neurochemical levels in the brain of zebrafish: focus on a few alcohol related targets. Physiology and Behavior, 2012 107: p. 773-780. [PubMed: 22313674] 


\section{Research Highlights}

- Zebrafish were exposed to dopamine antagonists during development

- Exposures altered larval behavior in a light-dark locomotor test

- Adult locomotor behavior in a predator escape assay was also abnormal

- $\quad$ Other adult behavioral assays did not appear affected

- A second strain of fish was differentially affected in the larval assay 

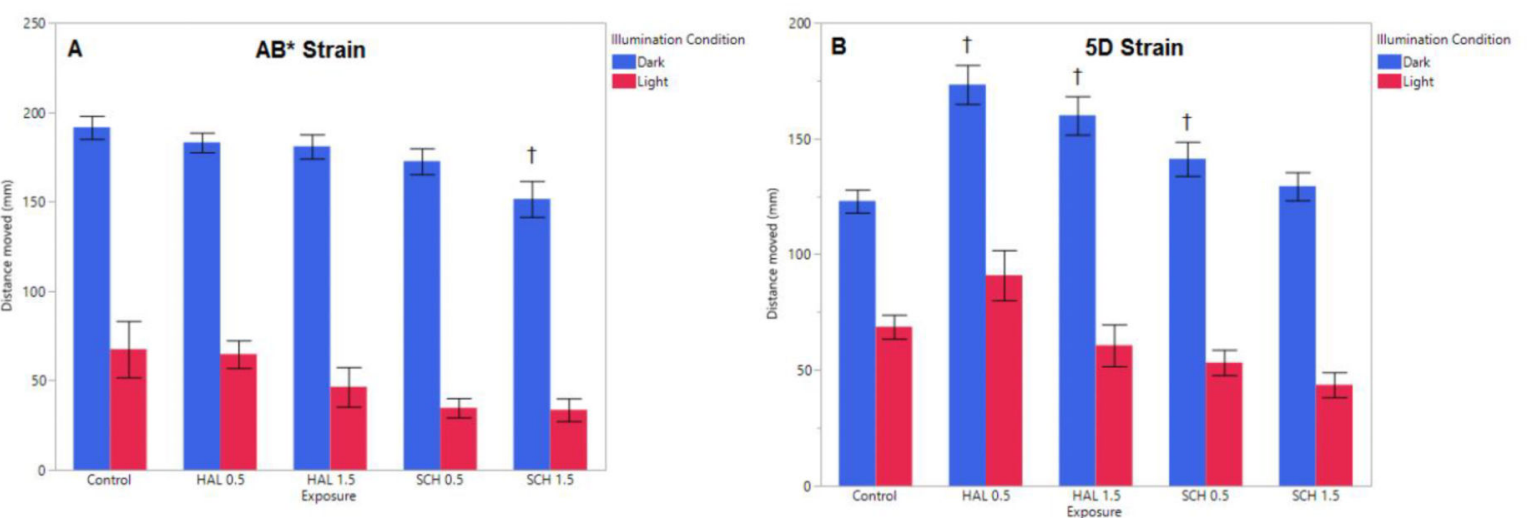

Figure 1.

Larval motility assay haloperidol and SCH-23390 dose effect functions in $\mathrm{AB}^{*}$ and $5 \mathrm{D}$ zebrafish strains red bars $=$ lit phase, blue bars $=$ dark phase $($ Mean \pm sem $)$ 


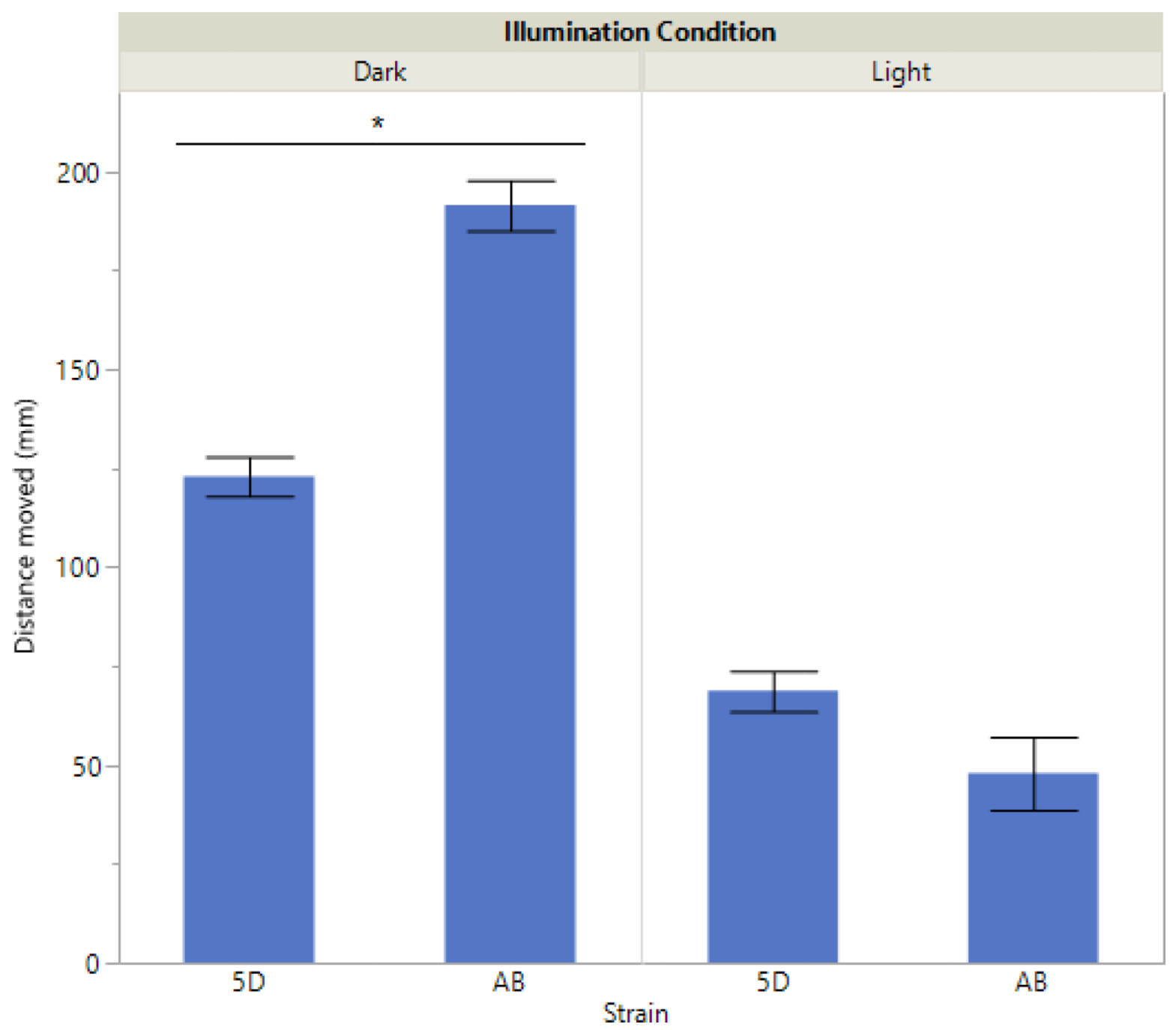

Figure 2.

Larval motility assay comparison of activity of vehicle treated control fish in each of the two strains $\left(\mathrm{AB}^{*}\right.$ and $\left.5 \mathrm{D}\right)$ during the lit and dark phases of testing (Mean \pm sem) 

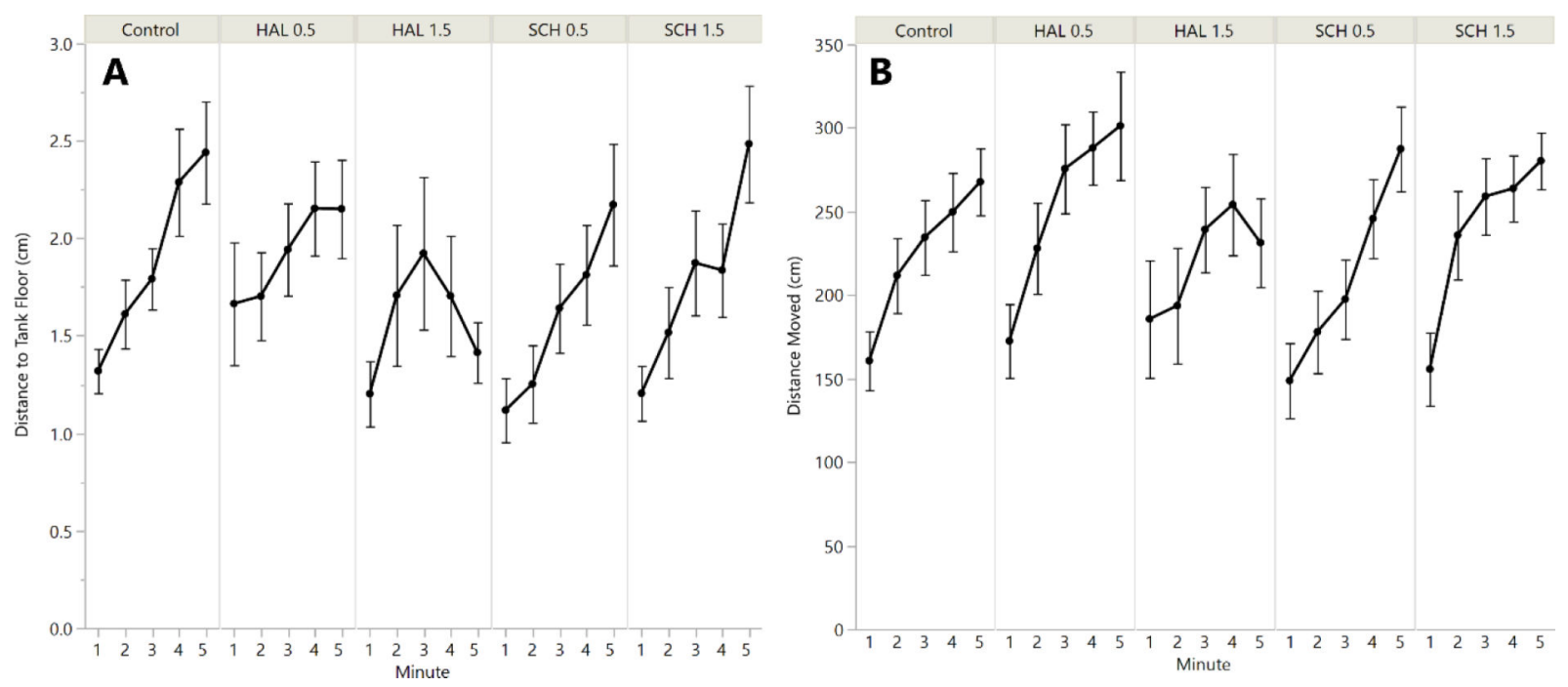

Figure 3.

Novel Tank Diving Test in the AB* zebrafish strain (Mean \pm sem) A) distance from the bottom of the tank and $\mathrm{B})$ swimming speed $(\mathrm{cm} / \mathrm{min})$. 

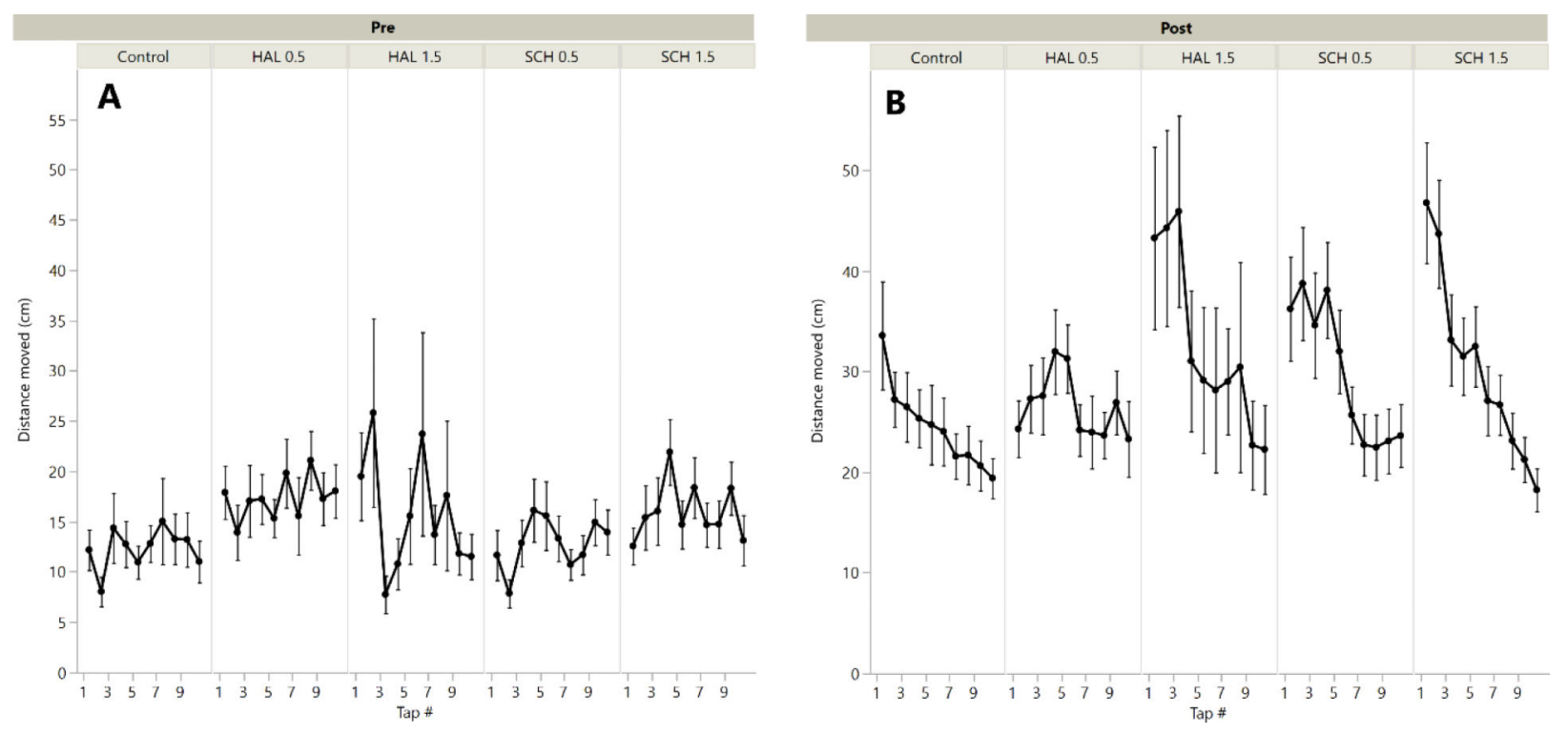

Figure 4.

Tap Startle Test distance moved (Mean \pm sem) A) 5-sec pre-tap, B) 5-sec post-tap over the series of ten taps at one-min intervals. 

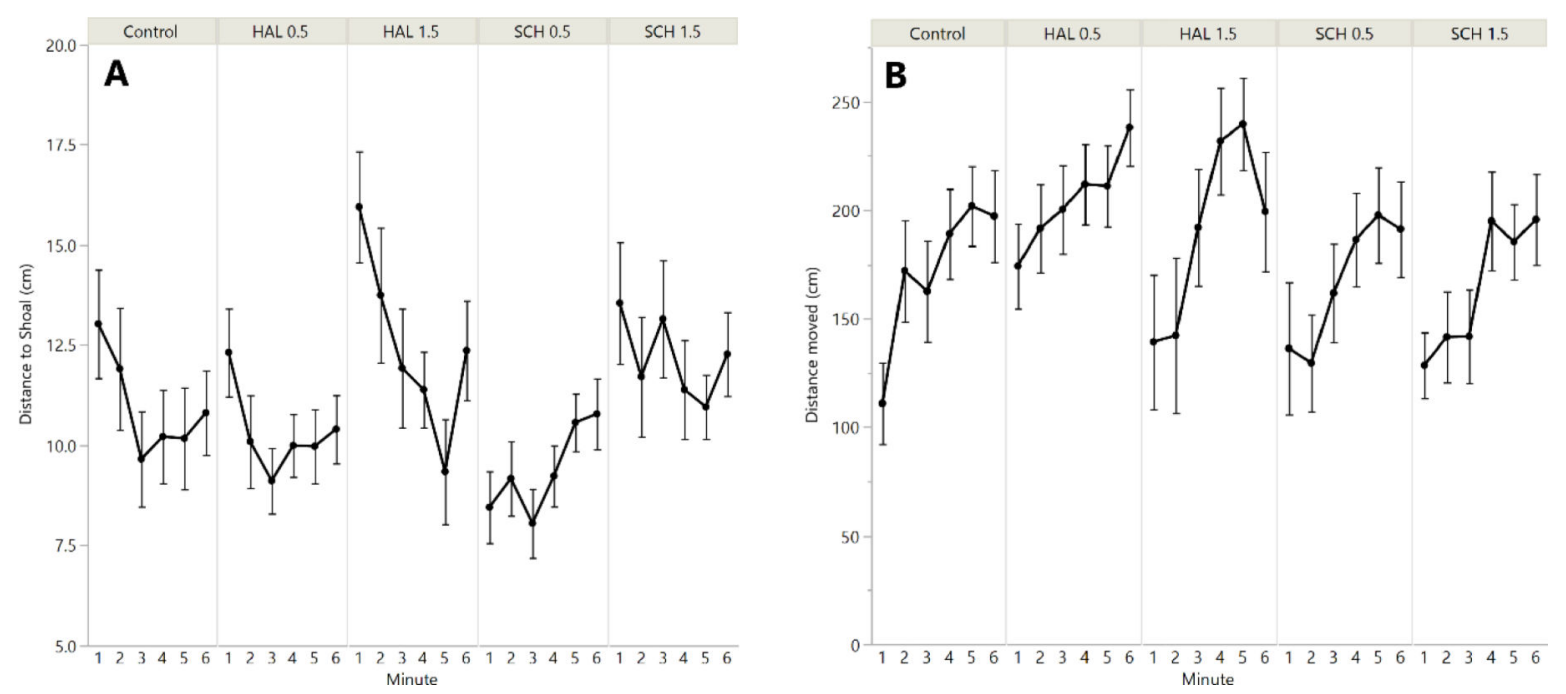

Figure 5.

Shoaling Test measures, A) average distance to shoal for each min and B) swimming speed $(\mathrm{cm} / \mathrm{min})(\mathrm{Mean} \pm \mathrm{sem})$ 

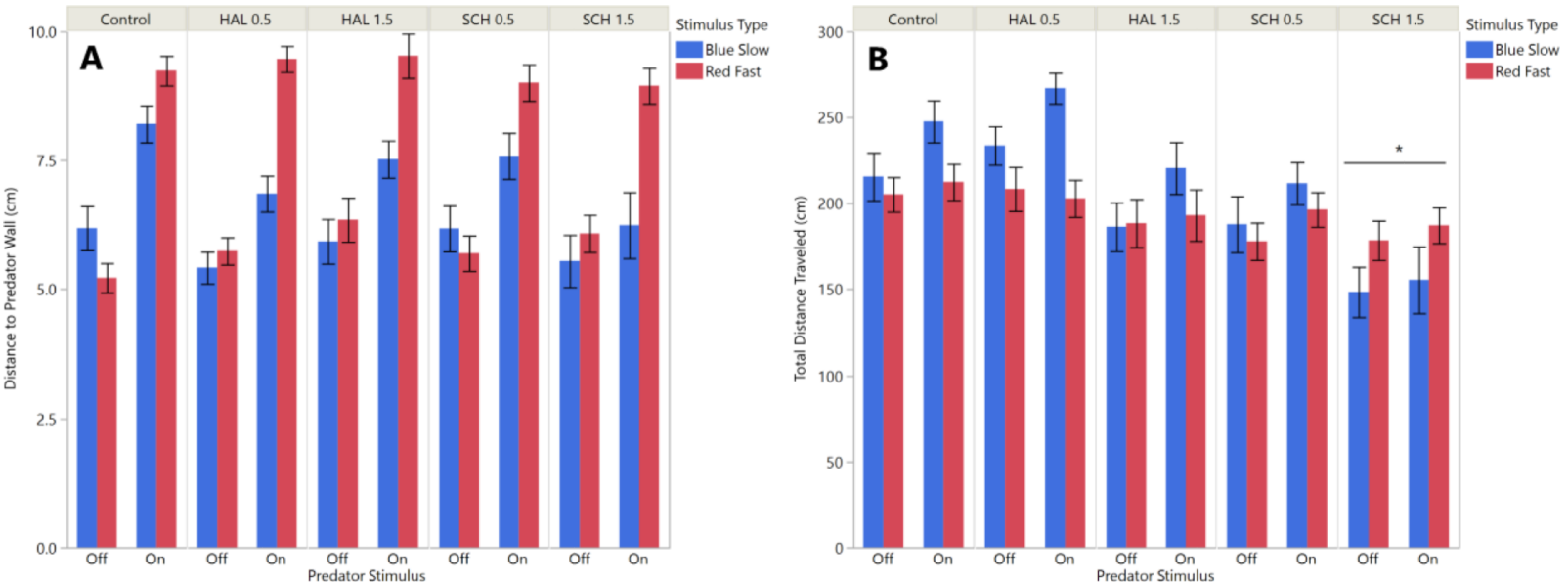

Figure 6.

Predator Avoidance Test measures the retreat of the zebrafish from enlarging red and blue visual stimuli. Two measures were taken A) distance from the stimulus and B) swimming speed $(\mathrm{cm} / \mathrm{min})($ Mean \pm sem) 